\title{
The Origin of Flaring Activity in AE Aquarii
}

Nazar Ikhsanov

Pulkovo Observatory, 196140 St. Petersburg, Russia

\section{Introduction}

\subsection{General properties}

AE Aqr is a close non-eclipsing binary system with an orbital period $P_{\text {orb }} \approx$ $9.88 \mathrm{hr}$, eccentricity of the orbit $e=0.02$ (Chincarini \& Walker 1981) and a mass ratio $q=1.14$ (Reinsch \& Beuermann 1994), situated at the distance of $\sim 84 \mathrm{pc}$ (Van Paradijs et al. 1989). The secondary is a $\mathrm{K} 3$ red dwarf on or close to the main sequence (Bruch 1991). The primary cannot be observed directly. However, the stable photometric oscillations at a period of $33 \mathrm{~s}$ (and at half this period) in the optical (Patterson 1979), UV (Eracleous et al. 1994), X-rays (Patterson et al. 1980) and, probably, VHE $\gamma$-rays (Meintjes et al. 1992, Bowden et al. 1992) leave no doubt that the primary is a rapidly rotating magnetized compact object. The observed rate of deceleration of the primary rotation is $\dot{P}=5.64 \times 10^{-14} \mathrm{ss}^{-1}$ (De Jager et al. 1994), and its mass is $m_{1} \sin ^{3} i=(0.56 \pm 0.03) M_{\odot}$ (Reinsch \& Beuermann 1994). The lack of eclipses allows to put a lower limit to the mass of the primary $m_{1} \geq 0.62 M_{\odot}$.

AE Aqr emits detectable radiation in all parts of the electromagnetic spectrum. In radio and $\gamma$-rays it is a powerful non-thermal source. However, in the optical, UV and X-rays the radiation is likely to be thermal and can be well explained by gas accretion onto a compact star (Patterson 1979, Patterson et al. 1980). The observed impulse profile of the pulsating component (Eracleous et al. 1994) looks similar to that of the accreting X-ray pulsars.

\subsection{Flaring component}

The main peculiarity of AE Aqr is its extraordinary flaring activity. Active and quiet phases alternate at a time scale of a few hours without any noticeable transitions. The persistent characteristic of the flare is flickering, clearly showing up in the power spectra during the active phase in contrast to the quiescence (Bruch 1991).

A major part of the thermal energy release in the flares is radiated in the UV part of spectrum. The light curves of AE Aqr during the active phase show a 
great diversity of flaring events and non-trivial fine structure. This raises the the question how meaningful average characteristics of the flares are. The most commonly adopted classification of flares has been based on their spectral properties (Chincarini \& Walker 1981, Bruch 1991):

1. pure continuum flares,

2. pure emission lines flares,

3. combination of line and continuum radiation.

With respect to their temporal characteristics three types of flaring components can be distinguished:

A: An active phase with durations of 3-5 hr, rise times of 1-1.5 hr and amplitudes in the U-band $\Delta m \sim 1^{\mathrm{m}}-2^{\mathrm{m}}$ (Chincarini \& Walker 1981, Bruch 1991). An active phase can be considered as a superposition of flares of shorter duration with the following characteristics:

B: Medium time scale flares lasting 10-30 min with rise times of 2-10 min (Van Paradijs et al. 1989). Some flares show multi-component fine structure:

C: Short time scale flashes with duration of 2-10 min and rise time of few seconds (De Jager \& Meintjes 1993).

The state of flaring activity of AE Aqr does not correlate with orbital phase (Van Paradijs et al. 1989). As a rule, an active phase begins with a sequence of flares of type $\mathbf{B}$ with increasing amplitude.

Near the maximum of the active phase two independent groups detected $\gamma$ ray emission from AE Aqr with energies $\sim \mathrm{TeV}$ and the luminosity $L_{\gamma} \sim 1.2 \times$ $10^{32}$ ergs $^{-1}$ (Meintjes et al. 1992, Bowden et al. 1992). Modulation of detected $\gamma$-ray radiation with a period $P_{1 / 2}=16.5 \mathrm{~s}$ gives evidence that it is generated in the vicinity of the compact object in $\mathrm{AE}$ Aqr.

Observations in the UV (Eracleous et al. 1994) have shown that the amplitude of the $33 \mathrm{~s}$ oscillations depends weakly on the state of the system (active/quiet). Finally, Abada-Simon et al. (1993) reported about the flaring character of the radio emission from AE Aqr. However, there is no evidence for a correlation between radio and optical flares.

\section{The primary and the evolutionary status of AE Aqr}

According to the standard model, proposed by Patterson (1979) the primary in $A E A q r$ is a rapidly rotating magnetized white dwarf undergoing disk accretion. Hence, AE Aqr can be classified as an intermediate polar, belonging to the DQ Her group. However, recent observational data force us to doubt the validity of this model. As is shown by Ikhsanov (1995a) any attempt to explain the totality of observational data on AE Aqr in the framework of the standard model leads to a paradox: the white dwarf should be simultaneously in three different states: ejector, propeller and accretor. This is in obvious contradiction with the modern theory on evolution of close binary systems. At the same time, this paradox does not arise under the assumption that the primary of $\mathrm{AEAqr}$ is a neutron star. According to Ikhsanov (1995a) the state of the neutron star can be classified 
as accretor-propeller. A principal feature of the new model is a low mass loss rate of the secondary during the quiet state $\dot{M} \sim 10^{12} \mathrm{~g} \mathrm{~s}^{-1}$. This is close to the mean loss rate of the late type stars in the form of stellar wind (Pottasch 1970). Chincarini \& Walker (1981) and Bruch (1991) pointed out that the degree of the secondary filling its Roche lobe might be insufficient to realize the mechanism of matter outflow through the first Lagrangian point $L_{1}$. Thus, according to this picture the evolutionary status of AE Aqr is determined as a pre-low-mass X-ray binary pulsar (Ikhsanov 1995a).

According to calculations of Davies \& Pringle (1980), a quasi-spherical type of accretion should be realized in this system. That is why we will discuss further a scenario of flaring activity in AE Aqr in the framework of the model of quasispherical accretion onto a magnetized neutron star.

\section{Main points of a flare scenario for AE Aqr}

Bruch (1991) pointed out that the flaring behaviour of AE Aqr does not fit the model of flaring activity of the secondary component with respect to either its temporal or its energetic characteristics. Thus, the flares are more likely to be connected with a non-stationary accretion process. The reasons for this can be as follows:

- variations of the secondary mass loss rate,

- instabilities in the accretion flow,

- interaction of the accretion flow with the magnetosphere of the primary.

Taking into account the temporal characteristics of type $\mathbf{B}$ (see 1.2), Van Paradijs et al. (1989) suggested that the trigger of the flares is localized at the Alfvén surface of the primary and is not connected with disk instabilities. They proposed a model of "magnetospheric gating", assuming that the state of the primary is quasi-periodically changing from propeller to accretor and back. However, this model cannot explain the temporal characteristics of the active phase $\mathbf{A}$ as a whole (see 1.2) as well as spectral differences between flares.

Starting from a model of quasi-spherical accretion onto a neutron star instead of the standard (accreting white dwarf) model can shed new light on the problem of flaring activity in AE Aqr..

First of all, neither the surface of a neutron star nor the accretion column can be the source of the observed thermal energy release during a flare. Otherwise we should detect flares in hard X-rays, rather than optical or UV flares. Thus, we can assume that the flare energy release is localized at the Alfven surface of the neutron star or/and at the surface of the secondary. In both cases, a flare proves to be connected with changes in the atmosphere of the secondary. Really, in the binary system with practically circular orbit and quasi-spherical type of accretion quasi-periodical increase of the rate of accretion onto the Alfvén surface can be connected only with change of the mass loss rate of the secondary. If we exclude the mechanism of the secondary magnetic activity (Bruch 1991), we can suppose that the state of the secondary may be changed due to interaction of 
the secondary and the primary. Such interaction may occurr via the illumination of the secondary by the radiation of the neutron star.

Models connected with the irradiation of the secondary by UV and X-rays (Osaki 1985, Hameury et al. 1986) cannot work in AE Aqr because of the very low luminosity in these spectral regions (Eracleous et al. 1994; Patterson et al. 1980). At the same time, we can suppose that perturbation of the red dwarf atmosphere may occur due to the non-thermal mode of the neutron star energy release (accelerated particles and $\gamma$-rays). Under certain conditions, heating of the red dwarf atmosphere by the beam of accelerated protons $\left(E_{\mathrm{p}} \sim \mathrm{GeV}\right)$ results in the increase of the secondary mass loss rate up to $\dot{M} \sim 10^{-10} M_{\odot} \mathrm{yr}^{-1}$ on a dynamical time scale $\sim 100 \mathrm{~s}$ (Ikhsanov 1995b). Accretion of this gas onto the Alfvén surface of the neutron star leads to UV flare with the blackbody temperature $T_{\text {eff }} \sim 16000-18000 \mathrm{~K}$. The rise time of such a flare is of the order of $b / V_{\mathrm{ff}}(b) \sim 1-1.5 \mathrm{hr}$, where $b$ is the orbital separation, and $V_{\mathrm{ff}}(b)$ is the free-fall velocity at this distance.

The different spectral behaviour of flares in this case can be classified according to the values of the flux of accelerated particles at the surface of the red dwarf. This value depends on the relation between the main axes of the system and the degree of collimation of the particle beam. If the flux reaches a critical value $S \geq S_{\mathrm{cr}}$, the model of explosive evaporation and matter accretion onto the Alfvén surface will be realized. Otherwise $\left(S<S_{\mathrm{cr}}\right)$, the mass loss rate of the red dwarf does not change much, and the flares are very similar to the secondary processes of flares on flaring stars (Livshits et al. 1981). A detailed scenario of flares will be presented in a subsequent paper (Ikhsanov 1995b).

\section{References}

Abada-Simon M., et al., 1993, Ap.J. 406, 692

Bowden et al., 1992, Astroparticle Phys. 1, 47

Bruch A., 1991, A\&A 251, 59

Chincarini G., Walker M.F., 1981, A\&A 104,24

Davies R.E., Pringle J.E., 1980, MNRAS 191, 599

de Jager O.C., et al., 1994, MNRAS 267, 577

de Jager O.C., Meintjes P.J., 1993, A\&A 268, L1

Eracleous M. et al., 1994, Ap.J. 433, 313

Hameury J.M., et al., 1986, A\&A 162, 71

Ikhsanov N.R. 1995a, A\&A in press

Ikhsanov N.R., 1995b, in preparation

Livshits M.A., et al., 1981, Solar Phys. 73, 269

Meintjes P.J., et al., 1992, ApJ 401, 325

Osaki Y., 1985, A\&A 144, 369

Paradijs J. van et al., 1989, A\&AS 79, 205

Patterson J., 1979, ApJ 234, 978

Patterson J., et al., 1980, ApJ. 240, L133

Pottasch S.R., 1970 in Interstellar Gas Dynamics, H.J. Habing (ed.), Reidel, Dordrecht Reinsch K., Beuermann K., 1994, A\&A 282, 493 